OPEN ACCESS

Edited by:

Mei-Ling Li,

Rutgers University, United States

Reviewed by:

Yueh-Hsin Ping,

National Yang-Ming University,

Taiwan

Victor R. DeFilippis,

Oregon Health \& Science University,

United States

*Correspondence:

Mukesh Kumar

mkumar8@gsu.edu

tThese authors have contributed equally to this work

Specialty section:

This article was submitted to

Virology,

a section of the journal

Frontiers in Microbiology

Received: 04 June 2019

Accepted: 26 August 2019 Published: 11 September 2019

Citation:

Rothan HA, Arora K, Natekar JP, Strate $P G$, Brinton MA and Kumar $M$ (2019) Z-DNA-Binding Protein 1 is

Critical for Controlling Virus Replication and Survival in West Nile Virus Encephalitis.

Front. Microbiol. 10:2089. doi: 10.3389/fmicb.2019.02089

\section{Z-DNA-Binding Protein 1 Is Critical for Controlling Virus Replication and Survival in West Nile Virus Encephalitis}

\author{
Hussin A. Rothan ${ }^{\dagger}$, Komal Arora ${ }^{\dagger}$, Janhavi P. Natekar, Philip G. Strate, Margo A. Brinton \\ and Mukesh Kumar*
}

Department of Biology, College of Arts and Sciences, Georgia State University, Atlanta, GA, United States

West Nile virus (WNV), a neurotropic flavivirus, is the leading cause of viral encephalitis in the United States. Recently, Zika virus (ZIKV) infections have caused serious neurological diseases and birth defects, specifically Guillain-Barrè syndrome and microcephaly. Z-DNA binding protein 1 (ZBP1) is a cytoplasmic sensor that that has been shown to play a significant role in initiating a robust immune response. We previously reported that WNV and ZIKV infections induce dramatic up-regulation of ZBP1 in mouse brains as well as in infected primary mouse cells. Herein, we show the critical role of ZBP1 in restricting the pathogenesis of WNV and ZIKV infections. Deletion of ZBP1 resulted in significantly higher morbidity and mortality after infection with a pathogenic WNV NY99 strain in mice. No mortality was observed in wild-type (WT) mice infected with the non-pathogenic WNV strain, Eg101. Interestingly, infection of ZBP1-/- mice with WNV Eg101 was lethal resulting in $100 \%$ mortality, suggesting that ZBP1 is required for survival after WNV infection. Viremia and brain viral load were significantly higher in ZBP1-/- mice compared to WT mice. In addition, protein levels of interferon (IFN)- $\alpha$, and inflammatory cytokines and chemokines were significantly higher in the serum and brains of infected ZBP1-/- mice compared to the WT mice. Primary mouse cortical neurons and mouse embryonic fibroblasts (MEFs) derived from ZBP1-/- mice produced higher virus titers compared to WT cells after infection with WNV NY99 and WNV Eg101. Similarly, neurons and MEFs lacking ZBP1 exhibited significantly enhanced replication of PRVABC59 (Asian) and MR766 (African) ZIKV compared to WT cells. The knockout of ZBP1 function in MEFs inhibited ZBP1-dependent virus-induced cell death. In conclusion, these data reveal that ZBP1 restricts WNV and ZIKV production in mouse cells and is required for survival of a peripheral WNV infection in mice.

Keywords: West Nile virus, flavivirus, Zika virus, Z-DNA-binding protein 1, DNA-dependent activator of IFN-regulatory factors, host-pathogen interaction, virus replication

\section{INTRODUCTION}

Members of the genus flavivirus are the most important arthropod-borne viruses causing disease in humans. West Nile virus (WNV) is a neurotropic flavivirus that infects humans, birds, and horses resulting in complex neurological sequelae (Brinton, 2013). WNV infection in humans is usually asymptomatic, but can cause severe neurological disease including meningitis, 
encephalitis, paralysis, and death (Brinton, 2013; Donadieu et al., 2013). Zika virus (ZIKV) infection can cause fever, headache, fatigue, and neurological symptoms. ZIKV infection is also associated with microcephaly in newborns and GuillainBarré syndrome in adults (Coyne and Lazear, 2016; Costa and Ko, 2018; Hygino da Cruz et al., 2018; Mehta et al., 2018; Rothan et al., 2018a,b). No anti-viral drugs currently exist for treating patients infected with WNV or ZIKV infection.

Z-DNA binding protein 1 (ZBP1), also called DAI, is one of the cytoplasmic DNA sensors that has been shown to play a significant role in initiating a robust immune response (Schwartz et al., 2001; Ha et al., 2006; Takaoka et al., 2007; Wang et al., 2008). Recent reports demonstrate that ZBP1 senses accumulation of RNA rather than DNA to initiate receptor-interacting protein homotypic interaction motif (RHIM)-dependent activation of receptor-interacting kinase-3 (RIPK3)-dependent necroptosis during HSV-1, murine cytomegalovirus virus (MCMV), influenza virus, and vaccinia virus infections (Kim et al., 2003; Upton et al., 2012; Pham et al., 2013; Wang et al., 2014; Kuriakose et al., 2016; Thapa et al., 2016; Kesavardhana et al., 2017; Koehler et al., 2017). Necroptosis is a form of cell death triggered by RIPK3 phosphorylation that activates the pseudo-kinase MLKL, which upon oligomerization ruptures the plasma membrane, leading to cell death (Wallach et al., 2016). Thus, necroptosis represents a host defense mechanism that combats virus replication in host tissues (Orozco and Oberst, 2017). In addition, recent work has implicated additional roles for ZBP1 and RIPK3 in promoting inflammation, independent of cell death (Daniels et al., 2017, 2019). ZBP1 also regulates NLRP3 inflammasome-mediated production of IL-1 $\beta$ in response to influenza virus infection (Kuriakose et al., 2016). In addition, ZBP1 has been shown to be involved in interferon (IFN) induction in response to HSV-1 (Wang et al., 2008) and human CMV infection (DeFilippis et al., 2010).

We previously reported that WNV and ZIKV infections induce dramatic up-regulation of ZBP1 in mouse brains as well as in infected primary mouse cells (Kumar et al., 2016; Azouz et al., 2019). In the present study, we show the critical role of ZBP1 in restricting the pathogenesis of $\mathrm{WNV}$ and $\mathrm{ZIKV}$ infections. The $\mathrm{ZBP}^{-/-}$mice exhibited higher morbidity and mortality after infection with lethal and non-lethal WNV strains compared to wild-type (WT) mice. Primary neuronal cultures and mouse embryonic fibroblasts (MEFs) lacking ZBP1 produced higher virus titers after infection with WNV and ZIKV compared to cells derived from WT mice. Collectively, these data provide the first evidence of the requirement for ZBP1 to restrict WNV and ZIKV production and demonstrate that ZBP1-dependent signaling is required to effectively control WNV infection in mice.

\section{MATERIALS AND METHODS}

\section{Animals}

Wild-type (WT) C57BL/6J mice were purchased from the Jackson Laboratory (Bar Harbor, ME), and $\mathrm{ZBP}^{-/-}$mice (nbio155) were obtained from the JCRB Laboratory Animal Resource Bank of the National Institutes of Biomedical Innovation, Health and
Nutrition (Osaka, Japan). All mice were bred and genotyped in the animal facility at Georgia State University. The WNV infection experiments were conducted in the animal biosafety level-3 laboratory. This study was carried out following the guidelines of the National Institutes of Health and the Institutional Animal Care and Use Committee (IACUC). The protocol was approved by the Georgia State University IACUC (Protocol number A19006).

\section{Animal Infection Experiments and Plaque Assay}

Eight-week-old WT and $\mathrm{ZBP}^{-/-}$mice were inoculated subcutaneously with 100 plaque-forming units (PFU) of WNV NY99, or 1,000 PFU of WNV Eg101, and the disease symptoms were observed twice daily (Kumar et al., 2013, 2014a,b; Krause et al., 2019). On specific days after inoculation, blood was collected from the tail vein, and serum was separated. In independent experiments, mice were inoculated with PBS (Mock) or WNV NY99 or WNV Eg101 subcutaneously, and on day 8 after inoculation, mice were anesthetized, extensively perfused with PBS, and the brains were harvested. WNV titers in the serum and brain homogenates were measured by plaque assay as described previously (Krause et al., 2019).

\section{West Nile Virus and Zika Virus Infection of Neuronal Cultures and Mouse Embryonic Fibroblast}

Mouse cortical neuron cultures and mouse embryonic fibroblasts (MEFs) were prepared from 1-day-old pups obtained from established colonies of $\mathrm{C} 57 / \mathrm{B} 6 \mathrm{~J} \mathrm{WT}$ and $\mathrm{ZBP}^{-1-}$ mice as described previously (Durkin et al., 2013; Forest et al., 2018; Azouz et al., 2019). The neurons were plated onto poly-Dlysine-coated 6-well or 24-well plates in serum Neurobasal-A medium (Gibco). The cultures were maintained in serum-free Neurobasal A medium supplemented with B27 (Gibco) for 7 days prior to infection. MEFs were grown in DMEM (Gibco) supplemented with $10 \%$ heat-inactivated fetal bovine serum and $10 \mu \mathrm{g} / \mathrm{ml}$ gentamicin (Gibco).

Primary neuronal cultures were infected with WNV NY99 at a multiplicity of infection (MOI) of 0.01 and MEFs were infected at a MOI of 1 . Both primary neuronal cultures and MEFs were infected with WNV Eg101 at a MOI of 1. For ZIKV infection experiments, neuronal cultures and MEFs were infected at a MOI of 1 with a ZIKV strain, PRVABC59 (Asian strain) or MR766 (prototype African strain). After infection, supernatants and cell lysates were harvested at 24, 48, and $72 \mathrm{~h}$ after infection. Virus titers were measured in cell supernatants by plaque assay (Kumar et al., 2013; Kim et al., 2018; Azouz et al., 2019).

\section{Enzyme-Linked Immunosorbent Assay and Multiplex Immunoassay}

Protein levels of IFN- $\alpha$ were measured in the serum and brain homogenates using the VeriKine ${ }^{\mathrm{TM}}$ Mouse Interferon- $\alpha$ enzymelinked immunosorbent assay (ELISA) Kit (PBL Interferon Source) as described previously (Kumar et al., 2012). Multiplex immunoassay kit (MILLIPLEX MAP Mouse Cytokine/Chemokine 
Kit, Millipore) was used to measure protein levels of inflammatory cytokines and chemokines in the serum (Kumar et al., 2012).

\section{Quantitative Reverse Transcription- Polymerase Chain Reaction}

Virus RNA levels were analyzed in the mouse brains and primary mouse cultures by quantitative reverse transcriptionpolymerase chain reaction (qRT-PCR). Briefly, total RNA was extracted from homogenized mice brains or cell lysates using a RNeasy Mini Kit (Qiagen) and a iScript ${ }^{\mathrm{TM}} \mathrm{cDNA}$ Synthesis Kit (Bio-Rad) was used to prepare cDNA samples. Quantitative RT-PCR was used to measure viral RNA levels using primers and probes specific for the WNV or ZIKV as described previously (Kumar et al., 2013, 2017).

\section{Cell Viability Assay}

Neuronal cultures and MEFs seeded in 96-well plates $\left(1 \times 10^{4}\right.$ cells/well) were mock-infected with PBS or infected with WNV NY99. Neuronal cultures were infected at a MOI of 0.01 , while MEFs were infected at a MOI of 1 . Cell viability was assessed at days 1-3 after infection using a CellTiter $96_{\mathrm{AQueous}}$ One Solution Cell Proliferation Assay (Promega) as described previously (Kumar et al., 2010).

\section{Statistical Analysis}

GraphPad Prism 7.0 was used to perform a Kaplan Meier log-rank test to compare survival curves. Unpaired Student's t-test using GraphPad was used to calculate values of $p$ for the clinical scores and plaque assay titers in mouse brains and serum. For plaque assay titers in cell culture supernatants and intracellular viral RNA copies in the cell lysates, two-way analysis of variance (ANOVA) with the post hoc Bonferroni test was used to calculate values of $p$. Differences with $p$ 's of $<0.05$ were considered significant.

\section{RESULTS}

\section{Z-DNA-Binding Protein 1 Signaling Controls West Nile Virus Pathogenesis in Mice Following Peripheral Infection}

To determine the role of ZBP1 in WNV pathogenesis, we evaluated morbidity of $\mathrm{WT}$ and $\mathrm{ZBP} 1^{-1-}$ mice after $\mathrm{WNV}$ infection. Mice were inoculated subcutaneously with either the lethal WNV, strain NY99 (100 PFU) or the non-lethal WNV, strain Eg101 (1,000 PFU). While the infectious dose of 100 PFU of WNV NY99 (Figure 1A) resulted in 40\% mortality in WT mice, mortality in $\mathrm{ZBP}^{-1-}$ mice was $100 \%$. The median survival time of infected $\mathrm{ZBP}^{-1-}$ mice was also shorter than in the WT mice. As expected, no mortality was observed in WT mice infected with 1,000 PFU of WNV Eg101 (Figure 1B). Interestingly, infection of $\mathrm{ZBP}^{-1-}$ mice with WNV Eg101 was highly lethal and resulted in $100 \%$ mortality. The median survival times observed in WNV Eg101-infected $\mathrm{ZBP}^{-1-}$ mice was similar to the WNV NY99-infected $\mathrm{ZBP}^{-/-}$mice.

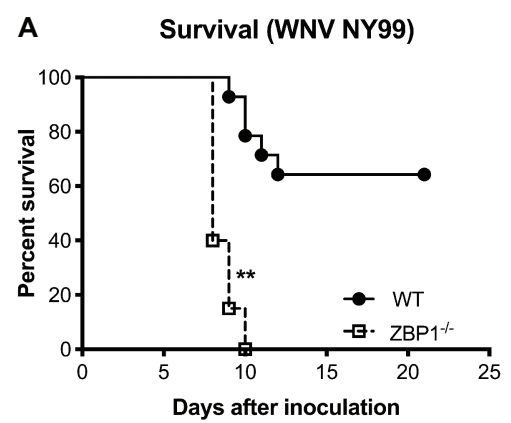

C

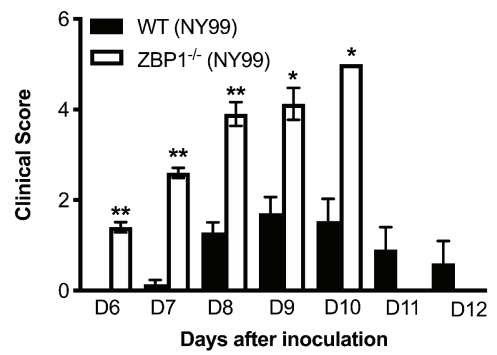

B

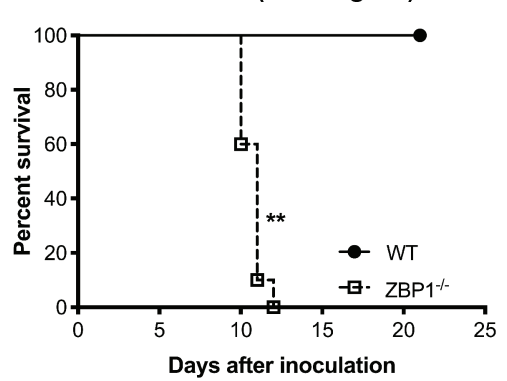

D

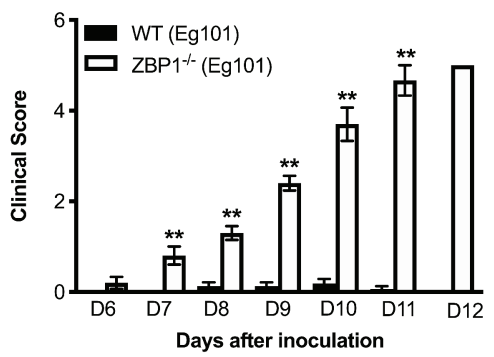

FIGURE 1 | Analysis of survival and clinical score in WT and ZBP1 $1^{-/-}$mice following WNV infection. WT and ZBP1 $1^{-/-}$mice were inoculated subcutaneously via footpad with (A) WNV NY99 (100 PFU) or (B) WNV Eg101 (1,000 PFU). The difference in the survival of WT and ZBP-/- mice was statistically significant for both WNV NY99 and WNV Eg101 ( $n=12-22$ mice per group). (C,D) Animals were monitored twice daily for clinical signs. The designation for the clinical scores is as follows: 1 , ruffled fur/hunched back; 2 , paresis/difficulty walking; 3, paralysis; 4, moribund/euthanized; and 5, dead. Error bars represent $\mathrm{SEM},{ }^{\star} p<0.05,{ }^{* \star} p<0.001$. 
All $\mathrm{ZBP}^{-/-}$mice developed severe neurological signs after inoculation with WNV NY99 or WNV Eg101 (Figures 1C,D). These clinical signs include ruffled fur, hunchbacked posture, paralysis, tremors, and ataxic gait. WT mice infected with WNV NY99 developed moderate clinical signs while the WT mice infected with Eg101 demonstrated no significant clinical signs. The observation of high morbidity and mortality in $\mathrm{ZBP}^{-/-}$mice inoculated with WNV Eg101 suggested that ZBP1 is required for survival after WNV infection in mice.

\section{Z-DNA-Binding Protein 1 Is Required for Control of West Nile Virus Load in the Periphery and Brain}

We next measured the viral titers in the serum of WT and $\mathrm{ZBP}^{-/-}$mice at different time-points after subcutaneous WNV NY99 or WNV Eg101 infection. The WNV replication kinetics in the serum of $\mathrm{WT}$ and $\mathrm{ZBP} 1^{-/-}$mice as measured by plaque assay demonstrated higher viremia in $\mathrm{ZBP}^{-/-}$mice. WNV titers were significantly higher in $\mathrm{ZBP}^{-/-}$mice as compared to WT mice at day 3 after WNV NY99 (Figure 2A) or WNV Eg101 infection (Figure 2B). At day 6 post-infection, WNV levels decreased in WT mice, while they remained significantly high in $\mathrm{ZBP}^{-/-}$mice infected with WNV NY99.

In separate experiments, mice were inoculated with WNV NY99 or WNV Eg101 subcutaneously, and brains were harvested at day 8 after inoculation. It is known that $\mathrm{WNV}$ is first detected in the mouse brain around day 6 after subcutaneous inoculation and peak virus load is observed at day 8 after infection. Therefore, we examined viral load in the brains at day 8 after infection. WNV titers in the brain homogenates were measured by plaque assay. WNV load in the brains of $\mathrm{ZBP}^{-/-}$mice was significantly higher than the WT mice infected with WNV NY99 or WNV Eg101 (Figure 2C). We next measured the WNV RNA copies in the brains of WT and $\mathrm{ZBP}^{-1-}$ mice infected subcutaneously with WNV NY99 or WNV Eg101. WNV RNA copies in the brains of $\mathrm{ZBP}^{-/-}$mice were significantly higher than the WT mice at day 8 after infection with WNV NY99 (Figure 2D). Very low levels of WNV RNA were detected in the brains of the WT mice infected with WNV Eg101. Nonetheless, significantly higher WNV RNA levels were detected in the brains of $\mathrm{ZBP}^{-1-}$ mice at day 8 after infection (Figure 2D). These data suggest that ZBP1-dependent signaling plays a significant role in controlling $\mathrm{WNV}$ load in both the periphery and in the brain.

\section{Anti-Viral Immune Responses in Wild-Type and $\mathrm{ZBP}^{-1-}$ Mice}

IFN- $\alpha$ is essential for the WNV clearance from the periphery and in the brain (Suthar et al., 2013). ZBP1 has also been shown to be involved in IFN induction after virus infection (Wang et al., 2008; DeFilippis et al., 2010). Therefore, we measured the protein levels of IFN- $\alpha$ in the serum (day 3 after infection) and brain homogenates (day 8 after infection) of $\mathrm{WT}$ and $\mathrm{ZBP}^{-/-}$mice using ELISA. Our data demonstrate
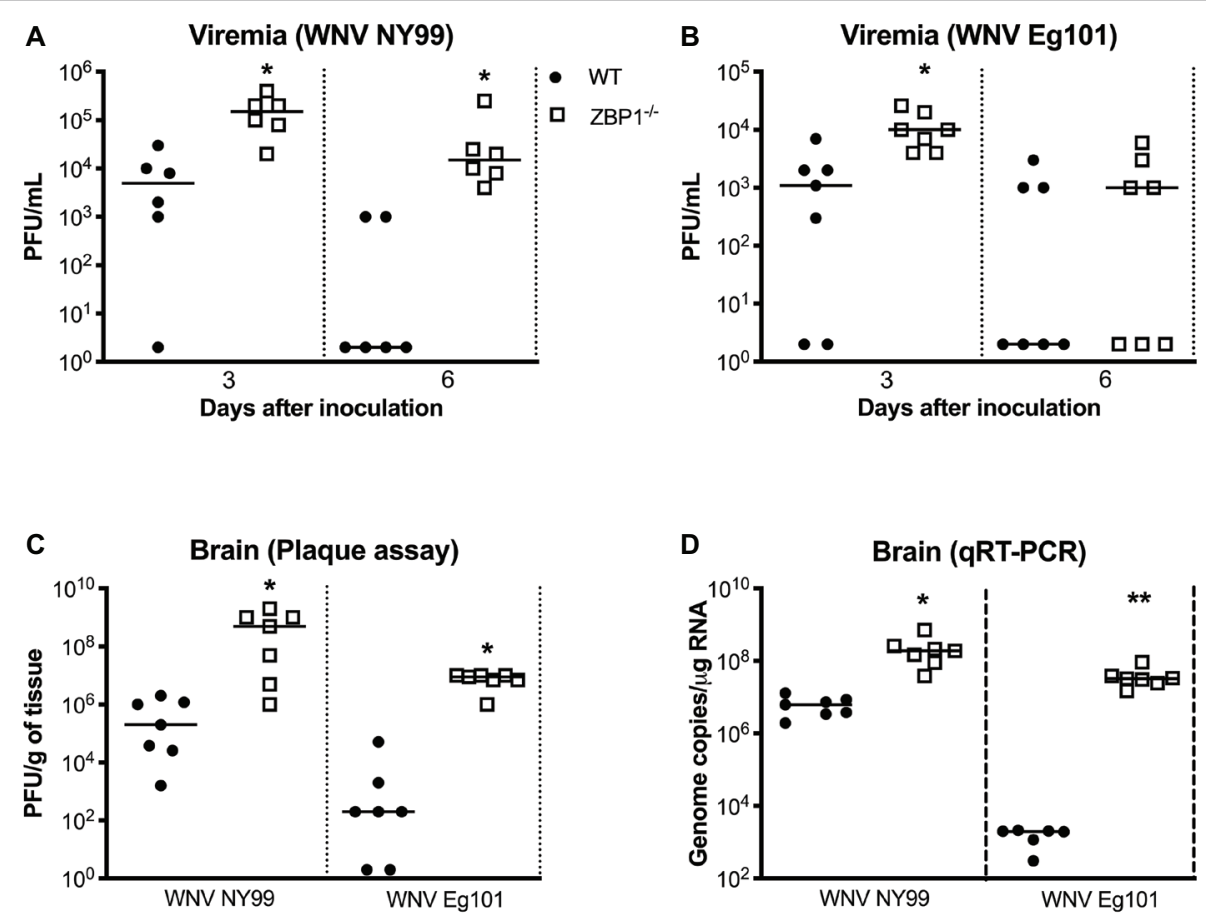

FIGURE 2 | Analysis of virus titers in WT and ZBP1-/- mice. Virus titers were measured in the serum at days 3 and 6 after (A) WNV NY99 or (B) WNV Eg101 infection by plaque assay and expressed as PFU/mL. (C) Virus titers were measured in brain homogenates (day 8 after infection with WNV NY99 or WNV Eg101) and expressed as PFU/g of tissue. (D) The WNV RNA copy number in the brain was determined by qRT-PCR and expressed as genome copies/ug of RNA (day 8 after infection with WNV NY99 or WNV Eg101). Each data point represents an individual mouse. The solid horizontal lines signify the median $(n=6-7$ mice per group). ${ }^{*} p<0.05,{ }^{* *} p<0.001$. 
that protein levels of IFN- $\alpha$ in the serum and brain homogenates were significantly higher in $\mathrm{ZBP}^{-/-}$mice compared to the WT mice (Figure 3).

Recent work has implicated a role for ZBP1 in promoting protective inflammation (Daniels et al., 2019). It is known that WNV infection induces a strong up-regulation of multiple cytokines and chemokines. WNV-induced pro-inflammatory mediators are also known to protect mice from lethal WNV disease (Suthar et al., 2013). Therefore, we next assessed the protein levels of key cytokines and chemokines in the serum using a multiplex immunoassay. Protein levels of key antiviral cytokines and chemokines were significantly higher in the serum of $\mathrm{ZBP}^{-/-}$mice compared to the WT mice. The protein levels of interleukin (IL)-1a, TNFa, MIG (CXCL9), and IP-10 (CXCL10) were significantly higher in $\mathrm{ZBP}^{-1-}$ mice compared to the WT mice at day 2 after infection (Figure 4).
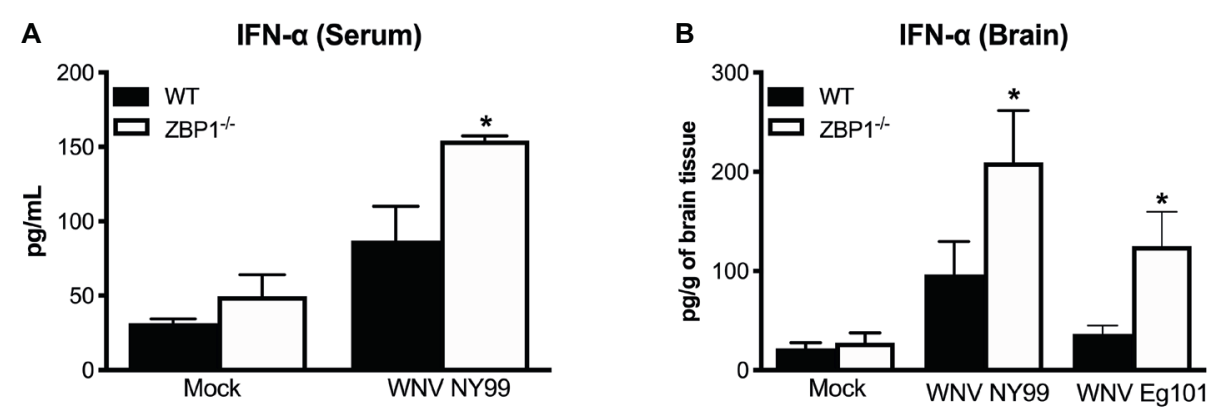

FIGURE 3 | IFN- $\alpha$ levels in the serum and brains of WT and ZBP1 ${ }^{-/-}$mice after infection with WNV NY99 or WNV Eg101. The protein levels of IFN- $\alpha$ were measured in the (A) serum and (B) brain homogenates using ELISA. Data represent the mean concentration $(\mathrm{pg} / \mathrm{ml}) \pm \mathrm{SEM}$ or $\left(\mathrm{pg} / \mathrm{g}\right.$ of tissue) $\pm \mathrm{SEM}\left(n=6-7 \mathrm{mice}\right.$ per group). ${ }^{*} p<0.05$.
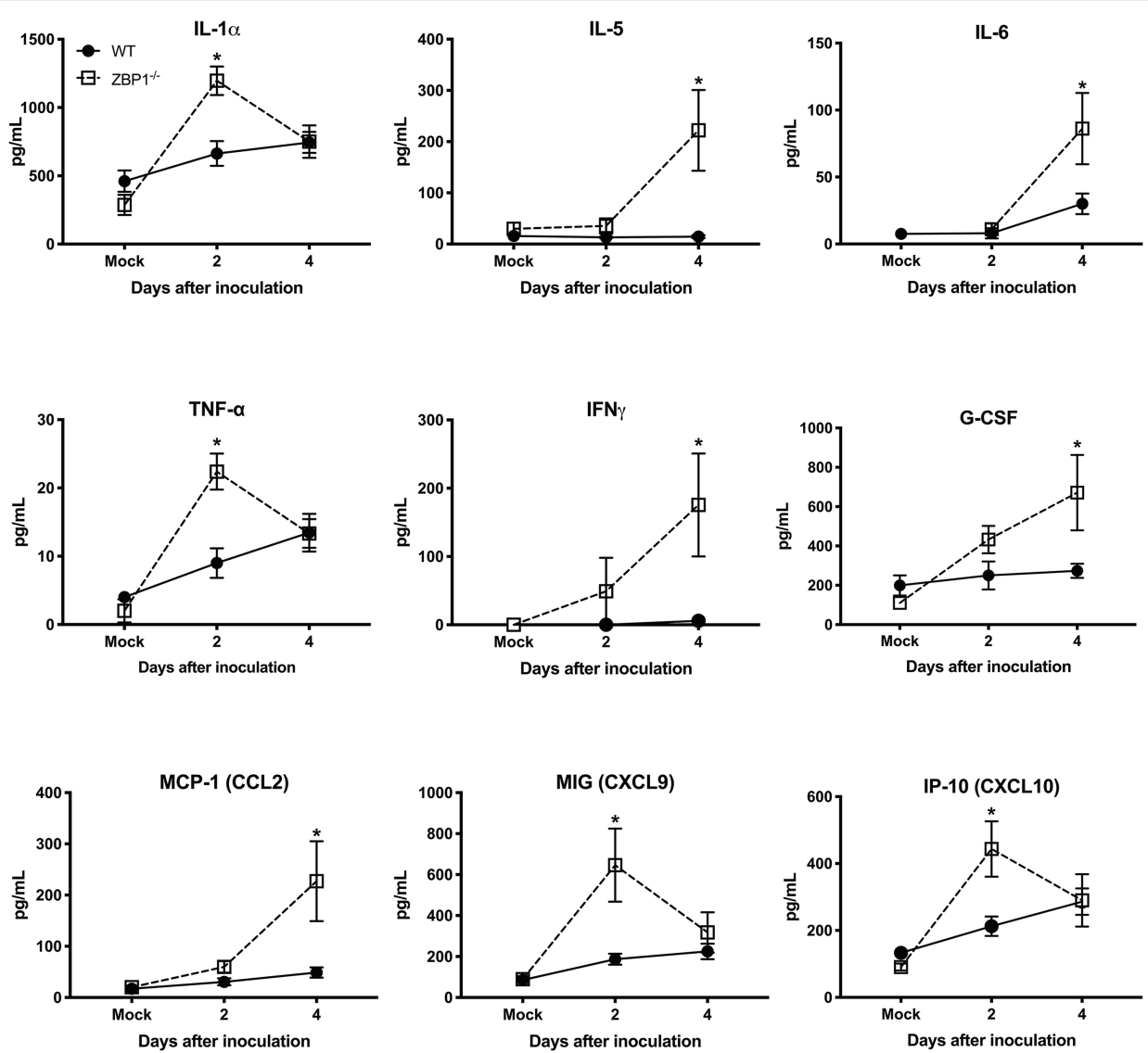

FIGURE 4 | Protein levels of cytokines and chemokines in the serum of WT and ZBP1-/- mice after infection with WNV NY99. Levels of cytokines and chemokines were measured in the serum of WNV NY99-infected WT and ZBP1 ${ }^{-/-}$at days 2 and 4 after infection. Data represent the mean concentration $(\mathrm{pg} / \mathrm{ml}) \pm \mathrm{SEM}(n=6-7$ mice per group). ${ }^{*} p<0.05$. 
The protein levels of IL-5, IL-6, IFN $\gamma$, G-CSF, and MCP-1 (CXCL2) were significantly higher in $\mathrm{ZBP}^{-/-}$mice compared to the WT mice at day 4 after infection (Figure 4). It is possible that high virus replication in $\mathrm{ZBP}^{-/-}$mice resulted in a higher inflammatory response. Collectively, these data indicate that ZBP1-mediated restriction of peripheral $\mathrm{WNV}$ infection is independent of IFN- $\alpha$, and anti-viral cytokines and chemokines.

\section{Z-DNA-Binding Protein 1 Restricts West Nile Virus and Zika Virus Replication in Mouse Cells}

To further define the role of ZBP1 during WNV infection, we performed a multistep virus growth analysis in cortical neurons and MEFs isolated from WT and $\mathrm{ZBP}^{-1-}$ mice. Cells were infected with WNV NY99 or WNV Eg101, and supernatants and cell lysates were harvested at 24,48 , and $72 \mathrm{~h}$ after infection. Virus titers were measured in cell supernatants by plaque assay. $\mathrm{WNV}$ infection of MEFs and neuronal cultures from $\mathrm{ZBP}^{-1-}$ mice resulted in significantly higher virus titers compared to those from WT mice (Figure 5). Total RNA was extracted from the cell lysates and WNV RNA copies were measured using qRT-PCR. Intracellular WNV RNA levels were also significantly higher in cell cultures from $\mathrm{ZBP}^{-/-}$mice compared to those from WT mice (Figure 6).
We next examined the role of ZBP1 in ZIKV replication. Similar to WNV, MEFs and neuronal cultures from $\mathrm{ZBP}^{-/-}$ mice produced significantly enhanced virus yields compared to those from WT mice after infection with the Asian or African strains of ZIKV (Figure 7). ZIKV RNA levels were also significantly higher in cell cultures from $\mathrm{ZBP}^{-/-}$mice compared to those from WT mice (Figure 8). The difference in both WNV and ZIKV titers between WT and $\mathrm{ZBP}^{-/-}$cells was consistently more dramatic in MEFs (2-3 logs) compared to cortical neurons $(1 \mathrm{log})$. These results correlate with the increased virus titers observed in the serum and brains of $\mathrm{ZBP}^{-1-}$ mice compared to the WT mice after WNV infection.

\section{Z-DNA-Binding Protein 1-Dependent Cell Death in Primary Mouse Cells Following Infection With West Nile Virus NY99}

$\mathrm{MEF}$ and neuronal cultures from $\mathrm{WT}$ and $\mathrm{ZBP}^{-/-}$mice were infected with WNV NY99 and cell viability was measured at 24,48 , and $72 \mathrm{~h}$ after infection. Our data demonstrate that the cell viability of infected $\mathrm{ZBP}^{-/-} \mathrm{MEF}$ was significantly higher than that of the WT MEFs at both 48 and $72 \mathrm{~h}$ after infection (Figure 9A). However, we did not observe this trend in neuronal cultures as the decrease in WNV-induced cell death was similar in both $\mathrm{WT}$ and $\mathrm{ZBP}^{-1-}$ neuronal cultures (Figure 9B).
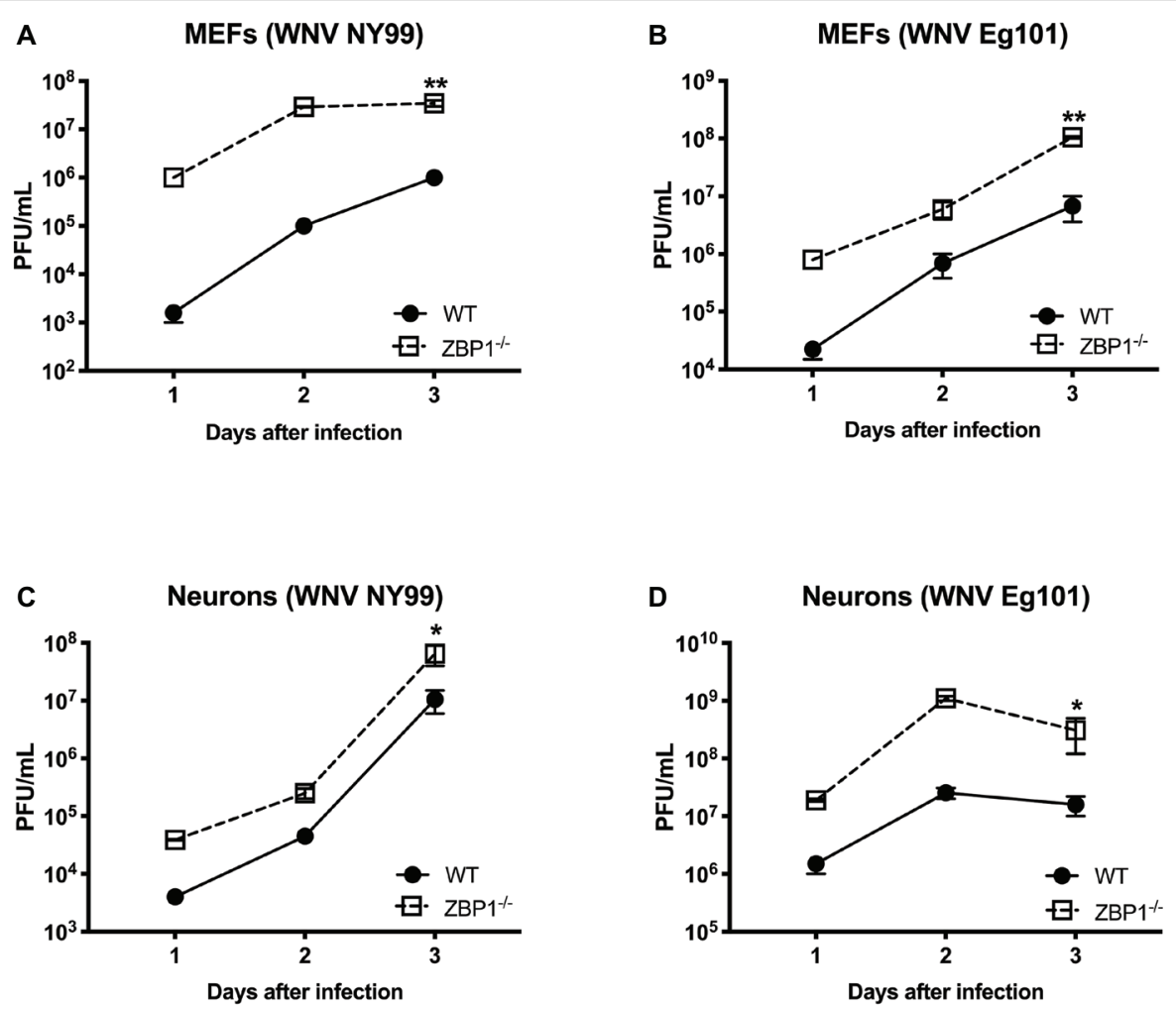

FIGURE 5 | Analysis of virus titers produced by primary cells isolated from WT and ZBP1-/- mice. (A-D) MEFs and neuronal cultures were infected with WNV NY99 or WNV Eg101 (as described in Materials and Methods) and virus titers in the cell culture supernatants were measured by plaque assay. Results are expressed as $\mathrm{PFU} / \mathrm{ml} \pm \mathrm{SEM}$ from at least three independent experiments conducted in duplicate. ${ }^{*} p<0.05,{ }^{* *} p<0.001$. 

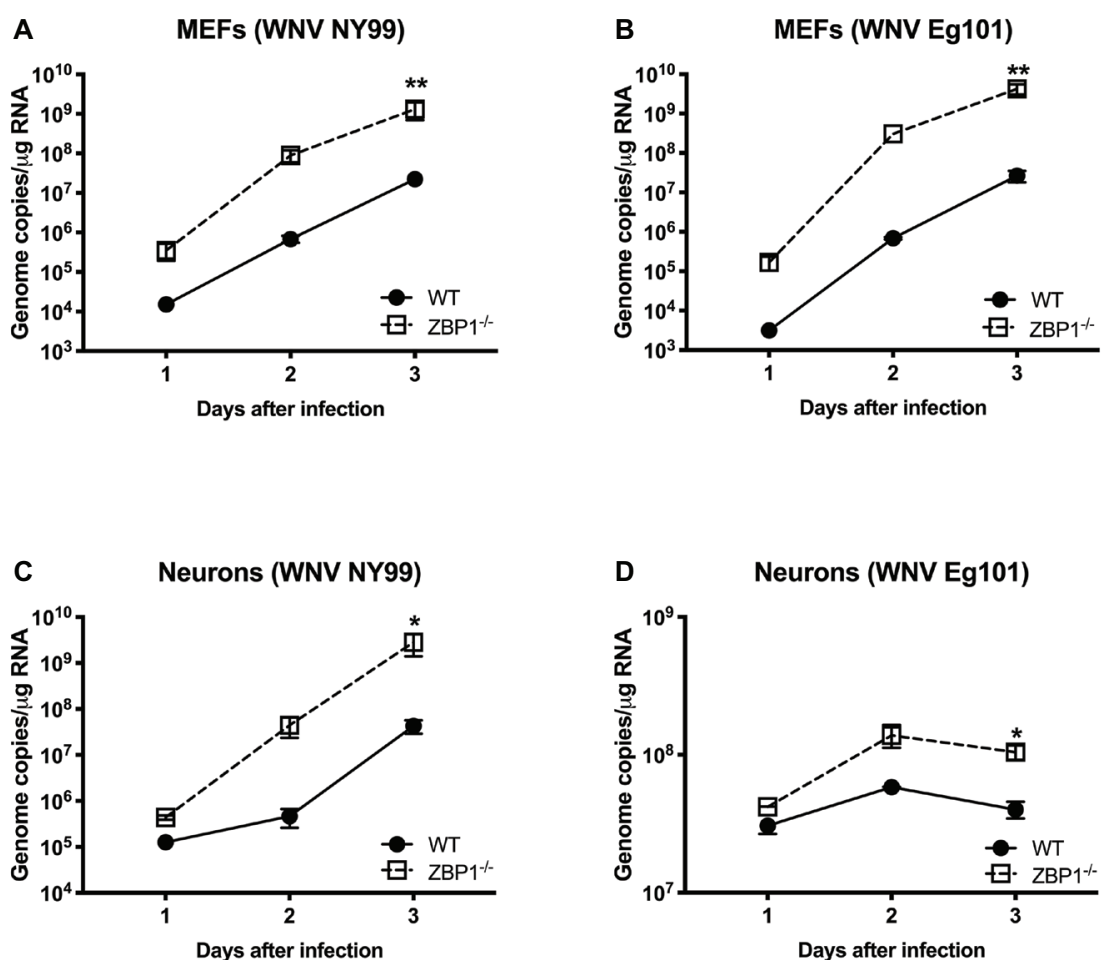

FIGURE 6 | Analysis of viral RNA levels in the primary cells isolated from WT and ZBP1-/- mice. (A-D) Cells were infected with WNV NY99 or Eg101 and total RNA extracted from cell lysates was used to conduct qRT-PCR to measure WNV RNA (expressed as genome copies/ $\mathrm{Mg}$ of RNA). Error bars represent SEM (three independent experiments conducted in duplicate), ${ }^{*} p<0.05,{ }^{* *} p<0.001$.
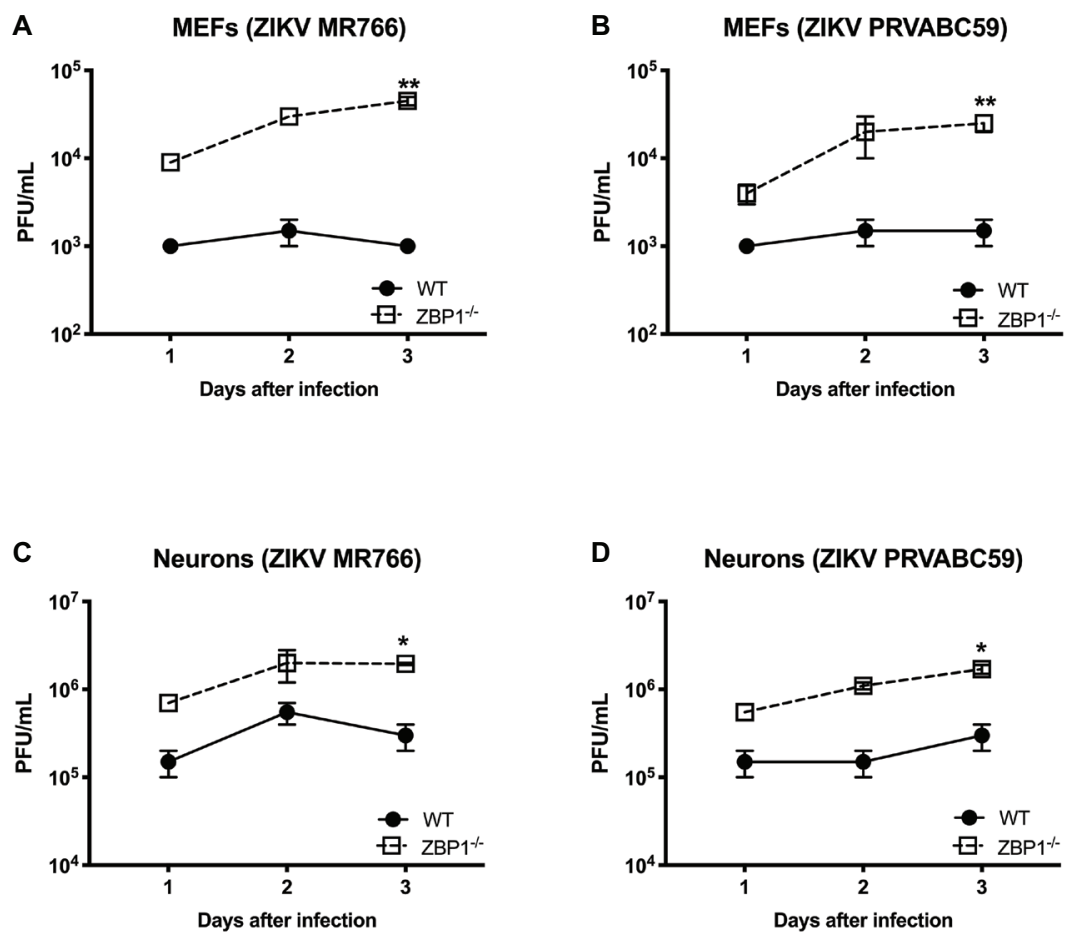

FIGURE 7 | Analysis of ZIKV yields produced by primary mouse cells. (A-D) MEFs and neuronal cultures derived from WT and ZBP1 ${ }^{-/-}$were infected with ZIKV MR766 or ZIKV PRVABC59 at a MOI of 1 and virus titers in the cell culture supernatants were measured by plaque assay. Results are expressed as PFU/mL \pm SEM from at least three independent experiments conducted in duplicate. ${ }^{*} p<0.05,{ }^{\star *} p<0.001$. 

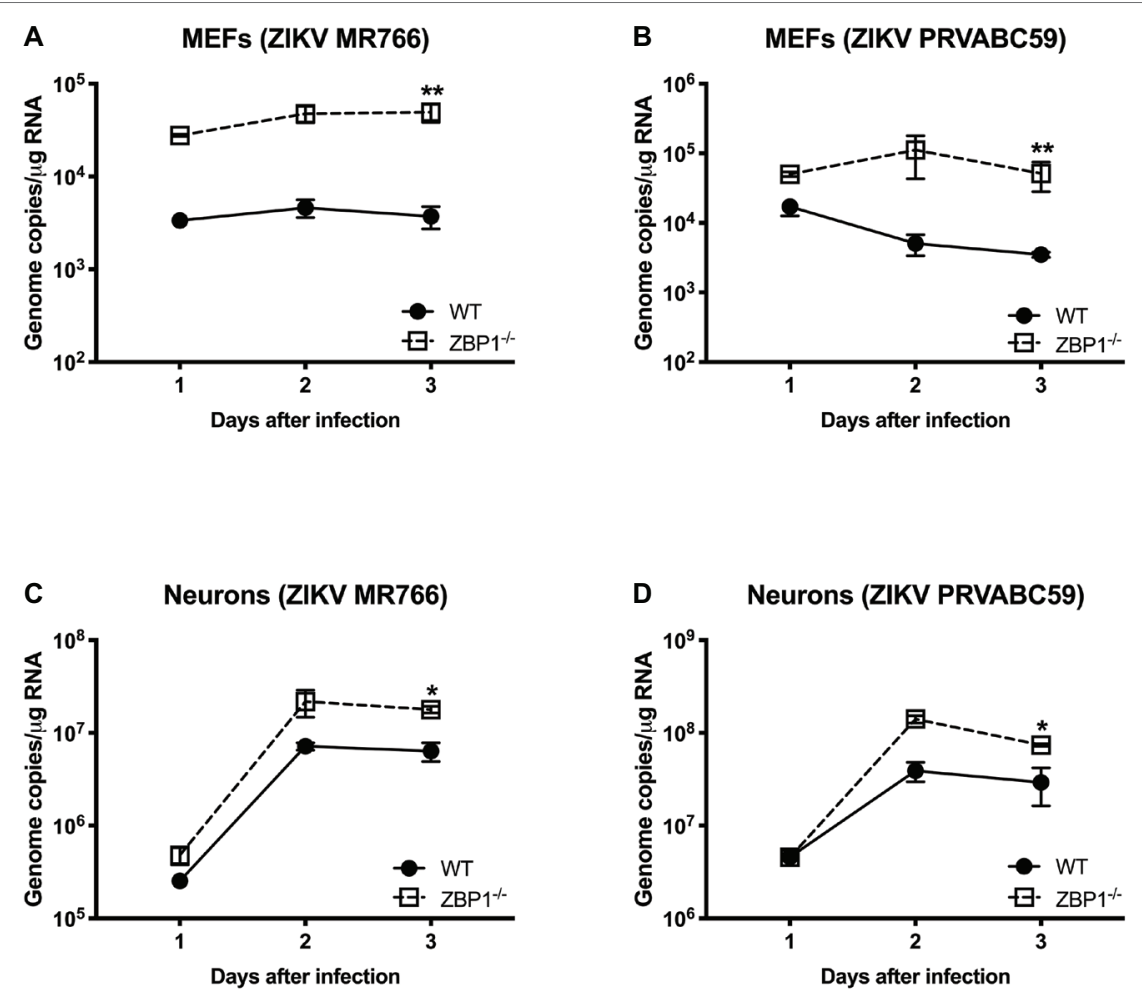

FIGURE 8 | ZIKV RNA levels in the MEFs and neurons isolated from WT and ZBP1-/- mice. (A-D) Cells were infected with ZIKV MR766 or ZIKV PRVABC59 at a MOI of 1. Total RNA was extracted from cell lysates and ZIKV RNA was measured by qRT-PCR (expressed as genome copies/ug of RNA). Error bars represent SEM (three independent experiments conducted in duplicate), ${ }^{*} p<0.05,{ }^{*} p<0.001$.
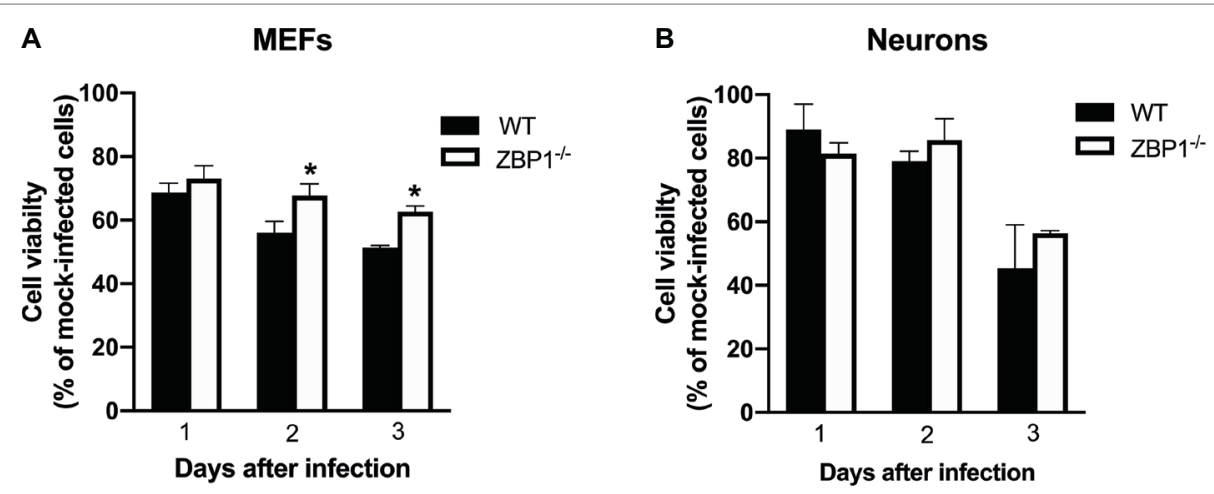

FIGURE 9 | Assay of primary mouse cell viability following WNV infection. (A) MEFs and (B) neuronal cultures from WT and ZBP1-/- mice were infected with WNV NY99. Cell toxicity on days 1,2, and 3 after infection was evaluated by cell proliferation assay and the percentage of cell viability was calculated by comparing values to those from mock-infected cells at the corresponding time points. The data are expressed as the mean \pm SEM for two independent experiments conducted in triplicate. Error bars represent SEM, ${ }^{*} p<0.05$.

\section{DISCUSSION}

Our data for the first time demonstrate the critical role of ZBP1 in restricting WNV-induced pathogenesis in mice. ZBP1 reduces WNV and ZIKV production in primary mouse cells and is crucial for survival in the mouse model of WNV disease.

It is known that WNV Eg101 is largely non-pathogenic in adult mice after subcutaneous inoculation (Shirato et al., 2004;
Kumar et al., 2014a). However, adult $\mathrm{ZBP1}^{-/-}$mice exhibited $100 \%$ mortality after subcutaneous inoculation of WNV Eg101. These data suggest a critical role for ZBP1 in controlling the pathogenic effects of a WNV infection. Viral loads were also significantly higher in the serum and brains of WNV-infected $\mathrm{ZBP}^{-1-}$ mice compared to those of the WT mice. Several previous studies have reported increased virus titers and disease severity in $\mathrm{ZBP}^{-/-}$mice after infection with influenza virus 
(Kuriakose et al., 2016; Thapa et al., 2016), HSV-1 (Guo et al., 2018), and MCMV (Upton et al., 2012; Sridharan et al., 2017). One recent study published while this manuscript was in preparation suggests that ZBP1 senses ZIKV infection and restricts disease pathogenesis after intracranial inoculation of ZIKV in mice (Daniels et al., 2019). Another study from the same group previously reported that neuronal RIPK3 signaling is required for survival after subcutaneous WNV infection in mice (Daniels et al., 2017). Our data are in agreement with these observations demonstrating that $\mathrm{ZBP} 1$ reduces $\mathrm{ZIKV}$ production in primary mouse cells. However, our data for the first time also demonstrate that ZBP1 restricts replication of $\mathrm{WNV}$ in mouse cells and is required for survival of a peripheral WNV infection in mice.

Recent work has implicated a role for ZBP1 in promoting protective inflammation. Daniels et al. reported that ZBP1mediated protective neuroinflammation is required for the protection against intracranial ZIKV infection (Daniels et al., 2019). In the present study, we showed that high virus titers in the serum of $\mathrm{ZBP}^{-/-}$mice were associated with elevated levels of anti-viral cytokines and chemokines after subcutaneous infection with WNV. Our results are in agreement with previous studies demonstrating high levels of pro-inflammatory cytokines and chemokines in $\mathrm{ZBP}^{-/-}$mice after infection with Toxoplasma gondii and influenza virus (Kuriakose et al., 2016; Pittman et al., 2016). It is known that IFN- $\alpha$ is essential for the WNV clearance from the periphery and in the brain (Suthar et al., 2013). ZBP1 has also been shown to be involved in IFN induction after virus infection (DeFilippis et al., 2010). Our data demonstrate that protein levels of IFN- $\alpha$ in the serum and brain homogenates were significantly higher in $\mathrm{ZBP}^{-/-}$ mice compared to the WT mice. Collectively, these data indicate that ZBP1-mediated restriction of peripheral WNV infection is independent of IFN- $\alpha$, and anti-viral cytokines and chemokines.

One interesting finding of our study was that we observed a dramatic difference in $\mathrm{WNV}$ or ZIKV virus replication in MEFs in the absence of ZBP1. In contrast, deletion of ZBP1 resulted in a modest increase in virus replication in neurons. In addition, we found that the viability of $\mathrm{ZBP}^{-1-} \mathrm{MEFs}$ infected with WNV was significantly higher than that of infected WT MEFs. However, the viability of $\mathrm{ZBP}^{-/-}$neurons was similar to that of WT neurons after WNV infection. These data are in agreement with previous observations demonstrating that ZIKV did not induce ZBP1-dependent cell death in primary neuronal cultures (Daniels et al., 2017,

\section{REFERENCES}

Azouz, F., Arora, K., Krause, K., Nerurkar, V. R., and Kumar, M. (2019). Integrated MicroRNA and mRNA profiling in Zika virus-infected neurons. Viruses 11:162. doi: 10.3390/v11020162

Brinton, M. A. (2013). Replication cycle and molecular biology of the West Nile virus. Viruses 6, 13-53. doi: 10.3390/v6010013

Costa, F., and Ko, A. I. (2018). Zika virus and microcephaly: where do we go from here? Lancet Infect. Dis. 18, 236-237. doi: 10.1016/S1473-3099(17)30697-7

Coyne, C. B., and Lazear, H. M. (2016). Zika virus - reigniting the TORCH. Nat. Rev. Microbiol. 14, 707-715. doi: 10.1038/nrmicro.2016.125
2019). However, ZBP1-dependent cell death in virus-infected MEFs had never been examined. It is known that activation of the ZBP1-RIPK3 pathway requires high levels of ZBP1 expression and therefore a cell-specific difference in the levels of ZBP1 expression may determine the outcome (Guo et al., 2018). Interestingly, it has been reported that the expression level of ZBP1 is strongly up-regulated in MEFs when stimulated with a synthetic DNA and therefore evokes a stronger innate immune response (Ishii et al., 2006). It could be possible that the ZBP1 and RIPK3 activation is more effective in MEFs compared to neurons. However, more studies are warranted to further understand the cell-specific role of ZBP1 in virus replication.

To our knowledge, our study for the first time revealed a critical role of ZBP1 during peripheral WNV infection in mice. There is need for further mechanistic studies to understand how ZBP1 restricts peripheral WNV and ZIKV infection.

\section{DATA AVAILABILITY}

All datasets generated for this study are included in the manuscript and/or the supplementary files.

\section{ETHICS STATEMENT}

The animal study was reviewed and approved by Georgia State University Institutional Animal Care and Use Committee (Protocol number A19006).

\section{AUTHOR CONTRIBUTIONS}

$\mathrm{MK}, \mathrm{HR}, \mathrm{KA}$, and $\mathrm{MB}$ designed the experiments, analyzed the data, and wrote the manuscript. MK, HR, KA, JN, and PS conducted the experiments. All authors have read and approved the final version of the manuscript.

\section{FUNDING}

This work was supported by a grant (R21NS099838) from National Institute of Neurological Disorders and Stroke and a grant (R21OD024896) from the Office of the Director, National Institutes of Health and Institutional funds.

Daniels, B. P., Kofman, S. B., Smith, J. R., Norris, G. T., Snyder, A. G., Kolb, J. P., et al. (2019). The nucleotide sensor ZBP1 and kinase RIPK3 induce the enzyme IRG1 to promote an antiviral metabolic state in neurons. Immunity 50, 64-76.e4. doi: 10.1016/j.immuni.2018.11.017

Daniels, B. P., Snyder, A. G., Olsen, T. M., Orozco, S., Oguin, T. H. III, Tait, S. W. G., et al. (2017). RIPK3 restricts viral pathogenesis via cell death-independent neuroinflammation. Cell 169, 301-313.e11. doi: 10.1016/j. cell.2017.03.011

DeFilippis, V. R., Alvarado, D., Sali, T., Rothenburg, S., and Fruh, K. (2010). Human cytomegalovirus induces the interferon response via the DNA sensor ZBP1. J. Virol. 84, 585-598. doi: 10.1128/jvi.01748-09 
Donadieu, E., Bahuon, C., Lowenski, S., Zientara, S., Coulpier, M., and Lecollinet, S. (2013). Differential virulence and pathogenesis of West Nile viruses. Viruses 5, 2856-2880. doi: 10.3390/v5112856

Durkin, M. E., Qian, X., Popescu, N. C., and Lowy, D. R. (2013). Isolation of mouse embryo fibroblasts. Bio. Protoc. 3:e908. doi: 10.21769/BioProtoc.908

Forest, K. H., Alfulaij, N., Arora, K., Taketa, R., Sherrin, T., Todorovic, C., et al. (2018). Protection against beta-amyloid neurotoxicity by a non-toxic endogenous $\mathrm{N}$-terminal beta-amyloid fragment and its active hexapeptide core sequence. J. Neurochem. 144, 201-217. doi: 10.1111/jnc.14257

Guo, H., Gilley, R. P., Fisher, A., Lane, R., Landsteiner, V. J., Ragan, K. B., et al. (2018). Species-independent contribution of ZBP1/DAI/DLM-1-triggered necroptosis in host defense against HSV1. Cell Death Dis. 9:816. doi: 10.1038/ s41419-018-0868-3

Ha, S. C., Van Quyen, D., Hwang, H. Y., Oh, D. B., Brown, B. A. II, Lee, S. M., et al. (2006). Biochemical characterization and preliminary X-ray crystallographic study of the domains of human ZBP1 bound to left-handed Z-DNA. Biochim. Biophys. Acta 1764, 320-323. doi: 10.1016/j.bbapap.2005.12.012

Hygino da Cruz, L. C. Jr., Nascimento, O. J. M., Lopes, F., and Da Silva, I. R. F. (2018). Neuroimaging findings of Zika virus-associated neurologic complications in adults. AJNR Am. J. Neuroradiol. 39, 1967-1974. doi: 10.3174/ajnr.a5649

Ishii, K. J., Coban, C., Kato, H., Takahashi, K., Torii, Y., Takeshita, F., et al. (2006). A toll-like receptor-independent antiviral response induced by doublestranded B-form DNA. Nat. Immunol. 7, 40-48. doi: 10.1038/ni1282

Kesavardhana, S., Kuriakose, T., Guy, C. S., Samir, P., Malireddi, R. K. S., Mishra, A., et al. (2017). ZBP1/DAI ubiquitination and sensing of influenza vRNPs activate programmed cell death. J. Exp. Med. 214, 2217-2229. doi: 10.1084/jem.20170550

Kim, Y. G., Muralinath, M., Brandt, T., Pearcy, M., Hauns, K., Lowenhaupt, K., et al. (2003). A role for Z-DNA binding in vaccinia virus pathogenesis. Proc. Natl. Acad. Sci. USA 100, 6974-6979. doi: 10.1073/pnas.0431131100

Kim, J. A., Seong, R. K., Kumar, M., and Shin, O. S. (2018). Favipiravir and ribavirin inhibit replication of Asian and African strains of Zika virus in different cell models. Viruses 10:72. doi: 10.3390/v10020072

Koehler, H., Cotsmire, S., Langland, J., Kibler, K. V., Kalman, D., Upton, J. W., et al. (2017). Inhibition of DAI-dependent necroptosis by the Z-DNA binding domain of the vaccinia virus innate immune evasion protein, E3. Proc. Natl. Acad. Sci. USA 114, 11506-11511. doi: 10.1073/pnas.1700999114

Krause, K., Azouz, F., Nakano, E., Nerurkar, V. R., and Kumar, M. (2019). Deletion of pregnancy zone protein and murinoglobulin-1 restricts the pathogenesis of West Nile virus infection in mice. Front. Microbiol. 10:259. doi: 10.3389/fmicb.2019.00259

Kumar, M., Belcaid, M., and Nerurkar, V. R. (2016). Identification of host genes leading to West Nile virus encephalitis in mice brain using RNA-seq analysis. Sci. Rep. 6:26350. doi: 10.1038/srep38916

Kumar, M., Krause, K. K., Azouz, F., Nakano, E., and Nerurkar, V. R. (2017). A guinea pig model of Zika virus infection. Virol. J. 14:75. doi: 10.1186/ s12985-017-0750-4

Kumar, M., O'connell, M., Namekar, M., and Nerurkar, V. R. (2014a). Infection with non-lethal West Nile virus Eg101 strain induces immunity that protects mice against the lethal West Nile virus NY99 strain. Viruses 6, 2328-2339. doi: $10.3390 / \mathrm{v} 6062328$

Kumar, M., Roe, K., Nerurkar, P. V., Namekar, M., Orillo, B., Verma, S., et al. (2012). Impaired virus clearance, compromised immune response and increased mortality in type 2 diabetic mice infected with West Nile virus. PLoS One 7:e44682. doi: 10.1371/journal.pone.0044682

Kumar, M., Roe, K., Nerurkar, P. V., Orillo, B., Thompson, K. S., Verma, S., et al. (2014b). Reduced immune cell infiltration and increased pro-inflammatory mediators in the brain of type 2 diabetic mouse model infected with West Nile virus. J. Neuroinflammation 11:80. doi: 10.1186/1742-2094-11-80

Kumar, M., Roe, K., Orillo, B., Muruve, D. A., Nerurkar, V. R., Gale, M. Jr., et al. (2013). Inflammasome adaptor protein apoptosis-associated speck-like protein containing CARD (ASC) is critical for the immune response and survival in West Nile virus encephalitis. J. Virol. 87, 3655-3667. doi: 10.1128/ JVI.02667-12

Kumar, M., Verma, S., and Nerurkar, V. R. (2010). Pro-inflammatory cytokines derived from West Nile virus (WNV)-infected SK-N-SH cells mediate neuroinflammatory markers and neuronal death. J. Neuroinflammation 7:73. doi: $10.1186 / 1742-2094-7-73$
Kuriakose, T., Man, S. M., Malireddi, R. K., Karki, R., Kesavardhana, S., Place, D. E., et al. (2016). ZBP1/DAI is an innate sensor of influenza virus triggering the NLRP3 inflammasome and programmed cell death pathways. Sci. Immunol. 1:aag2045. doi: 10.1126/sciimmunol.aag2045

Mehta, R., Soares, C. N., Medialdea-Carrera, R., Ellul, M., Da Silva, M. T. T., Rosala-Hallas, A., et al. (2018). The spectrum of neurological disease associated with Zika and chikungunya viruses in adults in Rio de Janeiro, Brazil: a case series. PLoS Negl. Trop. Dis. 12:e0006212. doi: 10.1371/journal.pntd.0006212

Orozco, S., and Oberst, A. (2017). RIPK3 in cell death and inflammation: the good, the bad, and the ugly. Immunol. Rev. 277, 102-112. doi: 10.1111/imr.12536

Pham, T. H., Kwon, K. M., Kim, Y. E., Kim, K. K., and Ahn, J. H. (2013). DNA sensing-independent inhibition of herpes simplex virus 1 replication by DAI/ZBP1. J. Virol. 87, 3076-3086. doi: 10.1128/JVI.02860-12

Pittman, K. J., Cervantes, P. W., and Knoll, L. J. (2016). Z-DNA binding protein mediates host control of toxoplasma gondii infection. Infect. Immun. 84, 3063-3070. doi: 10.1128/IAI.00511-16

Rothan, H. A., Bidokhti, M. R. M., and Byrareddy, S. N. (2018a). Current concerns and perspectives on Zika virus co-infection with arboviruses and HIV. J. Autoimmun. 89, 11-20. doi: 10.1016/j.jaut.2018.01.002

Rothan, H. A., Fang, S., Mahesh, M., and Byrareddy, S. N. (2018b). Zika virus and the metabolism of neuronal cells. Mol. Neurobiol. 56, 2551-2557. doi: 10.1007/s12035-018-1263-x

Schwartz, T., Behlke, J., Lowenhaupt, K., Heinemann, U., and Rich, A. (2001). Structure of the DLM-1-Z-DNA complex reveals a conserved family of Z-DNA-binding proteins. Nat. Struct. Biol. 8, 761-765. doi: 10.1038/nsb0901-761

Shirato, K., Kimura, T., Mizutani, T., Kariwa, H., and Takashima, I. (2004). Different chemokine expression in lethal and non-lethal murine West Nile virus infection. J. Med. Virol. 74, 507-513. doi: 10.1002/jmv.20205

Sridharan, H., Ragan, K. B., Guo, H., Gilley, R. P., Landsteiner, V. J., Kaiser, W. J., et al. (2017). Murine cytomegalovirus IE3-dependent transcription is required for DAI/ZBP1-mediated necroptosis. EMBO Rep. 18, 1429-1441. doi: 10.15252/ embr.201743947

Suthar, M. S., Diamond, M. S., and Gale, M. Jr. (2013). West Nile virus infection and immunity. Nat. Rev. Microbiol. 11, 115-128. doi: 10.1038/nrmicro2950

Takaoka, A., Wang, Z., Choi, M. K., Yanai, H., Negishi, H., Ban, T., et al. (2007). DAI (DLM-1/ZBP1) is a cytosolic DNA sensor and an activator of innate immune response. Nature 448, 501-505. doi: 10.1038/nature06013

Thapa, R. J., Ingram, J. P., Ragan, K. B., Nogusa, S., Boyd, D. F., Benitez, A. A., et al. (2016). DAI senses influenza a virus genomic RNA and activates RIPK3-dependent cell death. Cell Host Microbe 20, 674-681. doi: 10.1016/j.chom.2016.09.014

Upton, J. W., Kaiser, W. J., and Mocarski, E. S. (2012). DAI/ZBP1/DLM-1 complexes with RIP3 to mediate virus-induced programmed necrosis that is targeted by murine cytomegalovirus vIRA. Cell Host Microbe 11, 290-297. doi: 10.1016/j.chom.2012.01.016

Wallach, D., Kang, T. B., Dillon, C. P., and Green, D. R. (2016). Programmed necrosis in inflammation: toward identification of the effector molecules. Science 352:aaf2154. doi: 10.1126/science.aaf2154

Wang, Z., Choi, M. K., Ban, T., Yanai, H., Negishi, H., Lu, Y., et al. (2008). Regulation of innate immune responses by DAI (DLM-1/ZBP1) and other DNA-sensing molecules. Proc. Natl. Acad. Sci. USA 105, 5477-5482. doi: 10.1073/pnas. 0801295105

Wang, X., Li, Y., Liu, S., Yu, X., Li, L., Shi, C., et al. (2014). Direct activation of RIP3/MLKL-dependent necrosis by herpes simplex virus 1 (HSV-1) protein ICP6 triggers host antiviral defense. Proc. Natl. Acad. Sci. USA 111, 15438-15443. doi: $10.1073 /$ pnas. 1412767111

Conflict of Interest Statement: The authors declare that the research was conducted in the absence of any commercial or financial relationships that could be construed as a potential conflict of interest.

Copyright $\odot 2019$ Rothan, Arora, Natekar, Strate, Brinton and Kumar. This is an open-access article distributed under the terms of the Creative Commons Attribution License (CC BY). The use, distribution or reproduction in other forums is permitted, provided the original author(s) and the copyright owner(s) are credited and that the original publication in this journal is cited, in accordance with accepted academic practice. No use, distribution or reproduction is permitted which does not comply with these terms. 